This is the peer reviewed version of the following article:

Lempke, M., Keller, R., and Gerlinger, P. (2015)

Influence of spatial discretization and unsteadiness on the simulation of rocket combustors. Int. J. Numer. Meth. Fluids, 79: 437-455,

which has been published in final form at

http://dx.doi.org/10.1002/fld.4059.

This article may be used for non-commercial purposes in accordance with Wiley Terms and Conditions for Self-Archiving. 


\title{
Influence of Spatial Discretization and Unsteadiness on the Simulation of Rocket Combustors
}

\author{
Markus Lempke, Roman Keller*, and Peter Gerlinger \\ Institut für Verbrennungstechnik der Luft- und Raumfahrt, Universität Stuttgart, Pfaffenwaldring 38-40, 70569 Stuttgart
}

\section{SUMMARY}

This paper investigates some important numerical aspects for the simulation of model rocket combustors. Precisely, 1.) a new high order discretization technique (MLP ${ }^{l d}$ multi-dimensional limiting process, low diffusion) is presented and compared to conventional second order schemes with different flux limiters. 2.) time accurate URANS (unsteady RANS) simulations are performed to assess possible improvements in comparison to steady-state RANS (Reynolds-averaged Navier-Stokes) simulations. 3.) fully three-dimensional simulations of an axisymmetric rocket combustor are compared to twodimensional axisymmetric ones. All studies are based on the PennState Preburner Combustor experiment which uses gaseous oxygen and hydrogen. This comprehensive study offers unique insight into how the mentioned numerical influence factors change the flow field, flame, and wall heat fluxes in the model rocket combustor. Because wall heat fluxes are known from the experiment only, numerical results are compared with LES of other authors, too. It will be shown, that the high order spatial discretization significantly improves the agreement with measured wall heat fluxes at low additional computational cost. In general the transition from simple to more complex numerical approaches steadily improves the qualitative agreement between simulation and experiment. Copyright (c) 2015 John Wiley \& Sons, Ltd.

Received ..

KEY WORDS: high order discretization, MLP, multi-dimensional limiting process, rocket combustion, URANS, turbulence, coaxial injector 


\section{INTRODUCTION}

Some key aspects for the numerical simulation of rocket combustion chambers are reliable predictions of the developing flow field, the location of the flame, pressure oscillations, and the thermal wall heat load. Up to now experimental investigations $[1,2]$ are the most trusted approach in rocket combustor development. However, due to the high pressure levels even model combustor tests are very expensive and the exact replication of flight conditions in ground test facilities is difficult to achieve. Another disadvantage is the lack of available data from the interior of the combustor due to the challenges for optical diagnostics to be used in high pressure environments [3]. This lack of data is a problem for code development, too. In addition, the number of available experimental test cases for code validation is very low. Most of these experiments offer limited data only (e.g. wall pressures, temperatures, and wall heat fluxes) $[1,4,5]$ but rarely comprehensive information about velocity, species and flame properties as they are known for low pressure laboratory flames [6].

In comparison to experiments numerical simulations have the advantage that a complete data set is obtained. On the other hand, the complex processes in rocket combustors require further research in several fields. Up to now, most scientific simulations of rocket combustors are performed for simple geometries only (e.g. single coaxial injectors) which strongly differ from full scale engines. Moreover, large discrepancies are observed between different numerical techniques employed for the same test case $[7,8]$ and the reasons for these deviations are often unknown. Thus it is important to isolate some of the major influence criteria and to perform an extensive analysis of their impact on the solution. In this paper fluid mechanical and numerical aspects (discretization, grid, unsteadiness) in rocket combustors are investigated while for example real gas effects $[9,10,11,12]$ are not considered.

Tucker et al. [7, 13] made one of the first attempts to asses the predictive capabilities of a number of academic CFD solvers. For this study the PennState Preburner Combustor (PSBC) was chosen which is a combustion chamber with a geometrically simple axisymmetric single coaxial

\footnotetext{
${ }^{*}$ Correspondence to: Roman Keller, Institut für Verbrennungstechnik der Luft- und Raumfahrt, Universität Stuttgart, Pfaffenwaldring 38-40, 70569 Stuttgart. E-mail: roman.keller@dlr.de 
injector $[1,4]$. Results from different research groups are compared in the cited papers which use different degrees of complexity in their numerical simulations (2D, 3D, RANS, LES). It is shown, that the predicted flow fields and subsequently the wall heat fluxes vary tremendously. An objective comparison of these results is difficult, because different numerical schemes, turbulence closures, combustion models, and grids have been used. Nonetheless fundamental differences between steady and unsteady simulations became apparent. There still is the question, whether a steady-state twodimensional simulation can adequately predict the inherently unsteady combustion phenomena occurring in this combustor. Different groups published contradicting conclusions on this topic. Riedmann et al. [14] have shown outstanding predictions of the wall heat flux using steady RANS simulations. They observed convergence problems on finer grids due to an unsteady flow behavior and therefore limited their investigations to coarse grids. Lian and Merkle [15] compared steady and unsteady simulations and observed major differences. While their steady-state simulation could not even reproduce the general trend of the wall heat flux, results could be considerably improved by changing to time accurate simulations. Masquelet and Menon [16] compare unsteady twodimensional axisymmetric LES with fully three-dimensional ones and discuss issues emerging from the imposed symmetry boundary condition of 2D simulations. They concluded that even unsteady 2D simulations (LES) suffer from significant shortcomings and are not able to correctly predict the wall heat fluxes.

In this paper we examine the influence of a new fifth order spatial discretization technique (multi-dimensional limiting process, low diffusion, $\operatorname{MLP}^{l d}$ ) $[17,18]$ on the flow behaviour in the PennState Preburner Combustor. Besides different orders of discretization, steady and time accurate simulations as well as two- and three-dimensional simulations are compared. All calculations are performed with the same code. Thus the same computational grid, time discretization, turbulence and combustion model, as well as numerical solver is used. To the knowledge of the authors this is the most comprehensive study of the PennState Preburner Combustor and the first one which uses a high order spatial discretization technique. 


\section{GOVERNING EQUATIONS AND NUMERICAL SCHEME}

\subsection{Governing Equations}

All results presented in this paper are obtained using the in-house TASCOM3D (Turbulent All Speed Combustion Multigrid) solver which has been developed for the simulation of reacting $[19,20,21,22,23]$ and non-reacting $[24,25,26,18]$ sub- and supersonic flows. The set of governing equations is given by

$$
\frac{\partial \mathbf{Q}}{\partial t}+\frac{\partial\left(\mathbf{F}-\mathbf{F}_{\nu}\right)}{\partial x}+\frac{\partial\left(\mathbf{G}-\mathbf{G}_{\nu}\right)}{\partial y}+\frac{\partial\left(\mathbf{H}-\mathbf{H}_{\nu}\right)}{\partial z}=\mathbf{S}
$$

where $t$ is the physical time and the conservative variable vector is

$$
\mathbf{Q}=\left[\bar{\rho}, \bar{\rho} \tilde{u}, \bar{\rho} \tilde{v}, \bar{\rho} \tilde{w}, \bar{\rho} \tilde{E}, \bar{\rho} k, \bar{\rho} \omega, \bar{\rho} \sigma_{T}, \bar{\rho} \sigma_{Y}, \bar{\rho} \tilde{Y}_{i}\right]^{t}, \quad i=1,2, \ldots, N_{k-1}
$$

The vectors $\mathbf{F}, \mathbf{G}$ and $\mathbf{H}$ specify the inviscid fluxes in $x$-, $y$-, and $z$-direction, respectively, and the corresponding viscous fluxes are denoted by the index $\nu$. The variables in $\mathbf{Q}$ are the Reynoldsaveraged density $\bar{\rho}$, the Favre-averaged velocities $\tilde{u}, \tilde{v}$, and $\tilde{w}$, and the total specific energy $\tilde{E}$. The $k$ - $\omega$ [27] turbulence model in use requires transport equations for the turbulent kinetic energy $k$ and the turbulence frequency $\omega=\epsilon / k$ ( $\epsilon$ is the dissipation rate of $k$ ). Moreover, transport equations are solved for the temperature variance $\sigma_{T}$ and the sum of the species mass fractions variance $\sigma_{Y}$.

Finally $\tilde{Y}_{i}$ are species mass fractions and $N_{k}$ is the number of species considered. The source vector

$$
\mathbf{S}=\left[0,0,0,0,0, S_{k}, S_{\omega}, S_{\sigma_{T}}, S_{\sigma_{Y}}, \bar{S}_{Y_{i}}\right]^{t}, \quad i=1,2, \ldots, N_{k-1}
$$

contains entries from turbulence and chemical reactions. 


\subsection{Numerical solver and models}

The set of governing equations (1) is solved using an implicit Lower-Upper Symmetric GaussSeidel (LU-SGS) [28, 29, 20] finite-volume algorithm. Finite-rate chemistry is employed to model combustion which is treated implicitly and fully coupled with the fluid motion. All results presented in this paper use the 8-species, 21-steps reaction mechanism of Ó Conaire et al. [30] which is validated for the pressure range of $0.05-87$ bar. Thus it is well suited for the experimental test case investigated (52 bar). The solver works on structured multi-block grids and is fully parallelized and vectorized. It shows an excellent performance on vector computers as well as on massively parallel architectures [31].

Time accurate simulations are performed by a second or third order backward differentiation formula (BDF) scheme. Therefore an additional derivative of Q with respect to the pseudo time $\tau$ is inserted into the discretized form of Eq. (1). The pseudo time $\tau$ is used for non-physical inner iterations needed to solve the non-linear set of equations for one physical time step $\Delta t$ by a Newton iteration (dual-time stepping scheme) [32]. For any physical time step the pseudo time $\tau$ is advanced until convergence of the inner iteration is achieved. In case of steady-state simulations the inner iteration cycle is dropped and the solution is advanced in time usually using local time stepping with constant CFL number for convergence acceleration. To calculate the inviscid fluxes the $\mathrm{AUSM}^{+}$-up flux vector splitting of Liou [33] is used. This requires primitive variables at the cell interfaces of the structured grid which are determined by a 5th order upwind biased MLPld scheme (see Sect. 2.3). The formal discretization order is only achieved on orthogonal grids and decreases in regions where the grid is not sufficiently smooth. The viscous fluxes are calculated by central differences. The turbulent Prandtl and Schmidt numbers are assumed to be 0.7 .

To account for turbulence-chemistry interaction, a multi-variate assumed PDF (probability density function) approach is employed [34, 21, 26], assuming statistical independence between species and temperature fluctuations. For the temperature PDF a clipped Gaussian distribution is assumed which is defined by mean temperature and temperature variance $\sigma_{T}$. The joint PDF of an arbitrary number of species mass fractions is described by a multi-variate $\beta$-distribution [34], 
defined by the species mean mass fractions and the sum of species mass fraction variances $\sigma_{Y}$. The second order moments $\sigma_{T}$ and $\sigma_{Y}$ are obtained from separate transport equations.

\subsection{Multi-Dimensional Limiting Process}

Because the high order MLP (multi-dimensional limiting process) discretization [17, 35] is relatively new, its most important points are given in this section. For details and proofs concerning the fifth order MLP ${ }^{\text {ld }}$ technique used in this paper see Ref. [18].

As to calculate the inviscid fluxes (using $\mathrm{AUSM}^{+}$-up [33]) through a cell interface, left $(L)$ and right $(R)$ interface values $\left(q^{L}\right.$ and $\left.q^{R}\right)$ are required. They are calculated by a high order (up to fifth order) upwind biased polynomial approach. With respect to a cell interface located at $i+1 / 2$ the averaged cell centre values of $q$ from $i-2, i-1, \ldots, i+3$ are chosen (three upstream and two downstream values). The coefficients for the polynomial approach are calculated in advance and take a non-equal grid spacing into account. Based on a number of slope ratios $r_{i}^{L}=\Delta q_{i+1 / 2} / \Delta q_{i-1 / 2}, r_{i}^{R}=\Delta q_{i-1 / 2} / \Delta q_{i+1 / 2}$ the functions $\beta(r)$ [17]

$$
\left.\begin{array}{r}
\beta_{i}^{L}=2 r_{i}^{L} /\left(1+r_{i}^{L}\right) \\
\beta_{i+1}^{R}=2 r_{i+1}^{R} /\left(1+r_{i+1}^{R}\right)
\end{array}\right\} \text { 2nd order van Leer [36] }
$$

are used by Kim et al. [17] to calculate the required interface values

$$
\begin{aligned}
& q_{i+1 / 2}^{L}=q_{i}+0.5 \max \left[0, \min \left(\alpha^{L}, \alpha^{L} r_{i}^{L}, \beta_{i}^{L}\right)\right] \Delta q_{i-1 / 2} \\
& q_{i+1 / 2}^{R}=q_{i+1}-0.5 \max \left[0, \min \left(\alpha^{R}, \alpha^{R} r_{i+1}^{R}, \beta_{i+1}^{R}\right)\right] \Delta q_{i+3 / 2}
\end{aligned}
$$

in a way, which fulfills the TVD constraint $\Phi(r)$ of Sweby [37]. In this equation an additional parameter $\alpha$ is introduced. For $\alpha=2$ any high order polynomial approach is filtered by the TVD constraint of Sweby only. However, Kim et al. [17] take a further step by allowing $1 \leq \alpha \leq 2$. In 
this way a subset of the TVD region, the MLP region, is defined. The parameter $\alpha$ performs a linear downscaling of the TVD region from its upper limit $\alpha=2$ to the more viscous lower limit $\alpha=1$, which corresponds to the minmod limiter [18]. For practical applications $0 \leq \alpha \leq 2$ is also possible without adding too much numerical dissipation [18]. Reason for the introduction of $\alpha$ is the need of a new parameter which will be used to avoid local extrema at the corner points of a volume. This stabilizes the numerical scheme and allows better results if discontinuities are oblique to the computational grid. Moreover, convergence is improved and MLP converges in cases where standard TVD limiters fail $[17,35,18]$. MLP uses information from diagonally located volumes and thus combines the different coordinate directions.

Basic point of the MLP technique is the calculation of the parameter $\alpha$, which is shortly explained now. In order to check if the recostruction of the interface values creates a local extremum at any corner of a volume of the structured grid, the eight (in 3D) corner values $q^{M L P}$ are calculated [17] by

$$
q_{i+\kappa_{x} / 2, j+\kappa_{y} / 2, k+\kappa_{z} / 2}^{M L P}=q_{i, j, k}+\frac{1}{2} \kappa_{x} \alpha_{x} \Delta q_{x}^{m m}+\frac{1}{2} \kappa_{y} \alpha_{y} \Delta q_{y}^{m m}+\frac{1}{2} \kappa_{z} \alpha_{z} \Delta q_{z}^{m m}
$$

with $\kappa_{x}, \kappa_{y}, \kappa_{z}= \pm 1$ using

$$
\Delta q^{m m}=\max \left[0, \min \left(1, r_{i}\right)\right] \Delta q_{i-1 / 2}
$$

in the $x$-, $y$-, and $z$-direction. $\Delta q^{m m}$ is the change from the cell centre to the cell interfaces if the minmod limiter is employed, and $\alpha_{x}, \alpha_{y}, \alpha_{z}$ are free MLP parameters in the corresponding coordinate directions. The eight corner values $q^{M L P}$ are limited according to [17]

$$
Q_{i+\kappa_{x} / 2, j+\kappa_{y} / 2, k+\kappa_{z} / 2}^{\min } \leq q_{i+\kappa_{x} / 2, j+\kappa_{y} / 2, k+\kappa_{z} / 2}^{M L P} \leq Q_{i+\kappa_{x} / 2, j+\kappa_{y} / 2, k+\kappa_{z} / 2}^{\max } .
$$

The lower and upper limits $Q^{\min }$ and $Q^{\max }$ are defined to be the minimum or maximum value out of the eight cell centres' values surrounding the corner [17]. From this condition $\alpha_{x}, \alpha_{y}$, and $\alpha_{z}$ 
are derived. If condition (9) is fulfilled, there is no local extremum at any corner of the volume. Only two corners of a volume [18] have to be checked for a maximum $\left(Q_{1}^{\max }\right.$ and $\left.Q_{2}^{\max }\right)$ and a minimum $\left(Q_{1}^{\min }\right.$ and $\left.Q_{2}^{\min }\right)$. The first corner to be checked for a maximum is $\mathbf{C}_{1}^{\max }$ located at $i \max , j \max , k \max$ and for a minimum $\mathbf{C}_{1}^{\min }$ located at imin, jmin, kmin. The corner indices are obtained from

$$
\begin{aligned}
& \frac{i \max }{\operatorname{simin}} \stackrel{\quad}{\frac{j \max }{j m i n}} \\
& \Delta q_{x}^{m m}<0 \quad i-\frac{1}{2} \quad i+\frac{1}{2} \quad \Delta q_{y}^{m m}<0 \quad j-\frac{1}{2} \quad j+\frac{1}{2} \\
& \text { kmax kmin } \\
& \Delta q_{z}^{m m}>0 \quad k+\frac{1}{2} \quad k-\frac{1}{2} \quad . \\
& \Delta q_{z}^{m m}<0 \quad k-\frac{1}{2} \quad k+\frac{1}{2}
\end{aligned}
$$

The indices of the second point to be checked for a maximum $\left(\mathbf{C}_{2}^{\max }\right)$ and for a minimum $\left(\mathbf{C}_{2}^{\min }\right)$, respectively, follow from

\begin{tabular}{ccc} 
& $\mathbf{C}_{2}^{\max }$ & $\mathbf{C}_{2}^{\min }$ \\
\hline$\left|\Delta q_{x}\right|<\min \left(\left|\Delta q_{y}\right|,\left|\Delta q_{z}\right|\right)$ & imin,jmax,kmax & imax, jmin, kmin \\
$\left|\Delta q_{y}\right|<\min \left(\left|\Delta q_{x}\right|,\left|\Delta q_{z}\right|\right)$ & imax,jmin, kmax & imin, jmax, kmin \\
$\left|\Delta q_{z}\right|<\min \left(\left|\Delta q_{x}\right|,\left|\Delta q_{y}\right|\right)$ & imax,jmax,kmin & imin, jmin, kmax
\end{tabular}.

The check of both corners is combined by the calculation of the maximum absolute change $\Delta q^{c}$ that is allowed to fullfill Eq. (9) [18]

$$
\begin{array}{r}
\Delta q^{c}=2 \min \left(Q_{1}^{\max }-q_{i, j, k}, Q_{2}^{\max }-q_{i, j, k}+2\left|\Delta q_{\min }^{m m}\right|,\right. \\
\left.q_{i, j, k}-Q_{1}^{\min }, q_{i, j, k}-Q_{2}^{\min }+2\left|\Delta q_{\min }^{m m}\right|\right) .
\end{array}
$$

Here $\left|\Delta q_{\min }^{m m}\right|=\min \left(\left|\Delta q_{x}^{m m}\right|,\left|\Delta q_{y}^{m m}\right|,\left|\Delta q_{z}^{m m}\right|\right)$ is the smallest absolute change from the three coordinate directions. 
While in one dimension there is a unique value of $\alpha$ to fullfill Eq. (9), this is not the case for two- or three-dimensional flows. In the $\operatorname{MLP}^{l d}$ version used in this paper $\alpha_{x}, \alpha_{y}, \alpha_{z}$ are chosen in order [18]

1. to fullfill Eq. (9) which is achieved by

$$
\left|\Delta q^{M L P}\right|=\alpha_{x}\left|\Delta q_{x}^{m m}\right|+\alpha_{y}\left|\Delta q_{y}^{m m}\right|+\alpha_{z}\left|\Delta q_{z}^{m m}\right|=\Delta q^{c}
$$

Note, that the equality sign (instead of $\leq$ as in Eq. (9)) is taken in order to obtain maximum $\alpha$ values and to minimize the restriction of the original discretization by MLP,

2. to keep the change in the mean gradient direction by the introduction of $\alpha_{x}, \alpha_{y}$, and $\alpha_{z}$ as small as possible.

$\left|\Delta q^{M L P}\right|=\Delta q^{c}=$ constant defines a limiting plane in the three-dimensional discretization space $\left|\Delta q_{x}\right|,\left|\Delta q_{y}\right|,\left|\Delta q_{z}\right|[18]$. Additional limitations are given by the TVD constraint. To keep the second condition given above, the absolute mean gradient direction has to be calculated what is done by second order central differences

$$
\begin{aligned}
\left|\Delta \bar{q}_{x}\right| & =\left|q_{i+1, j, k}-q_{i-1, j, k}\right|, \quad\left|\Delta \bar{q}_{y}\right|=\left|q_{i, j+1, k}-q_{i, j-1, k}\right| \\
\left|\Delta \bar{q}_{z}\right| & =\left|q_{i, j, k+1}-q_{i, j, k-1}\right|
\end{aligned}
$$

Next the coordinates of the point of intersection (point $\mathbf{A}$ ) between the line of the absolute mean gradient direction and the limiting plane $\left|\Delta q^{M L P}\right|=\Delta q^{c}=$ constant are calculated. The aim is to obtain a solution $\left|\Delta q^{M L P}\right|$ which corresponds as much as possible with the mean gradient direction. It is shown in Ref. [18] that this approach achieves the smallest possible reduction of $\alpha$ and thus remains as close as possible to the original high order discretization. Using the scaling factor

$$
f=\Delta q^{c} /\left(\left|\Delta \bar{q}_{x}\right|+\left|\Delta \bar{q}_{y}\right|+\left|\Delta \bar{q}_{z}\right|\right)
$$


the coordinates of the point $\mathbf{A}=\mathbf{A}\left(A_{x}, A_{y}, A_{z}\right)$ become

$$
A_{x}=f\left|\Delta \bar{q}_{x}\right|, \quad A_{y}=f\left|\Delta \bar{q}_{y}\right|, \quad A_{z}=f\left|\Delta \bar{q}_{z}\right| .
$$

If point $A$ is located inside the TVD region it directly defines the final $\alpha$ values. However, if $A$ is outside the TVD region defined by Sweby [37], it has to be moved on the plane $\left|\triangle q^{M L P}\right|=$ $\Delta q^{c}=$ constant until the required conditions are met. The corresponding procedure for MLP ${ }^{l d}$ with $\alpha_{x}, \alpha_{y}, \alpha_{z} \in[0,2]$, in combination with Eqs. (8) to (16) to initialise the variables, is:

$$
\operatorname{MLP}^{l d}\left\{\begin{aligned}
& \text { set } \alpha_{i}=2 \\
&\text { if } \left.\left(2\left(\left|\Delta q_{x}^{m m}\right|+\left|\Delta q_{y}^{m m}\right|\right)+\left|\Delta q_{z}^{m m}\right|\right)>\Delta q^{c}\right) \text { then } \\
& \text { do for } i=x, y, z \\
& h_{i}=\max \left(A_{i}-2\left|\Delta q_{i}^{m m}\right|, 0\right) \\
& U_{i}=A_{i}-h_{i}, \quad U_{j}=A_{j}+h_{i} / 2 \quad \text { for } j=x, y, z \text { and } j \neq i \\
& f=h_{i} /\left(h_{i}+\epsilon\right) \quad \text { with } \epsilon \text { is a small number } \\
& l= f\left[\max \left(A_{j}-2\left|\Delta q_{j}^{m m}\right|, 0\right)-\max \left(A_{k}-2\left|\Delta q_{k}^{m m}\right|, 0\right)\right] \\
& j, k=x, y, z \text { and } j, k \neq i \text { and } j \neq k \\
& S_{i}=U_{i}, \quad S_{j}=U_{j}-l, \quad S_{k}=U_{k}+l \\
& \alpha_{n}=S_{n} /\left|\Delta q_{n}^{m m}\right|, \quad A_{n}=S_{n} \quad \text { for } n=x, y, z \\
& \text { end do } \\
& \text { end if } \quad
\end{aligned}\right.
$$

The resulting $\alpha_{x}, \alpha_{y}$, and $\alpha_{z}$ values can be used in Eq. (6) in combination with any higher order approach for $\beta$. In some cases the final $\alpha$ values are already obtained after the first iteration of the loop over $x, y$, and $z$. In this case these values remain unchanged while performing the second and third iteration. The latter ones are needed because changes may occur in the second or third iteration, too. 


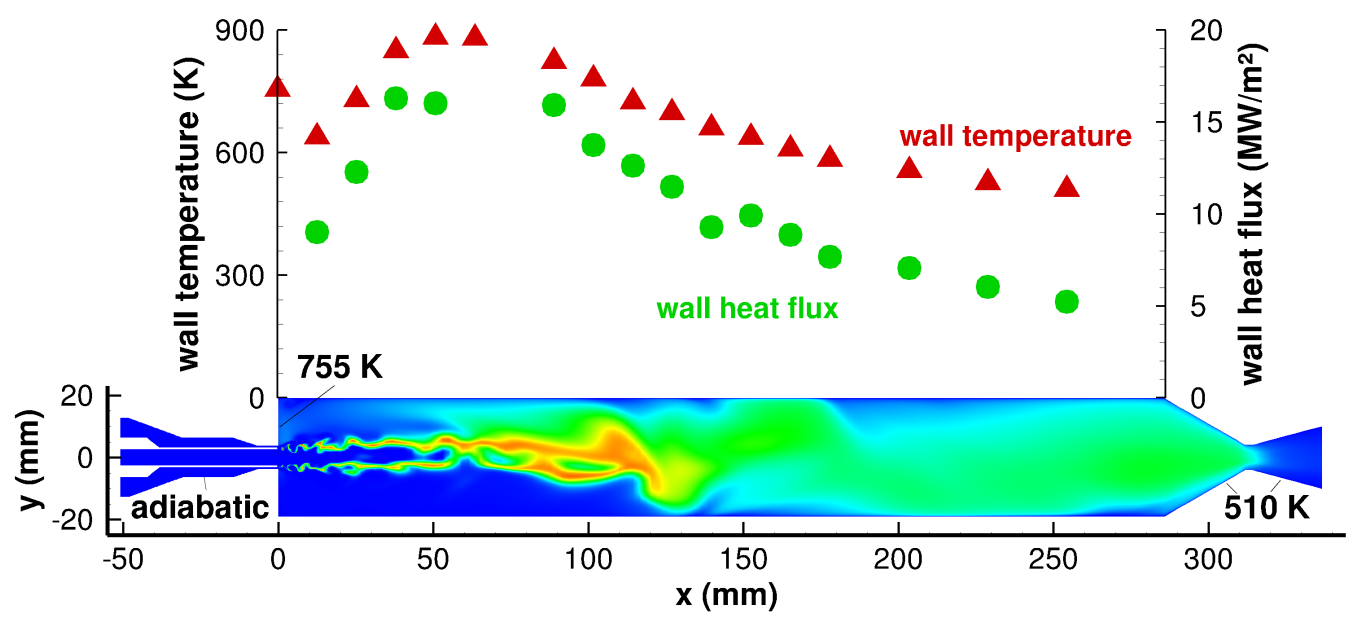

Figure 1. Geometry, boundary conditions, and measured wall temperatures (triangles) and wall heat fluxes (circles) for the PennState Preburner Combustor [1, 4]. Additionally a calculated instantaneous hydroxyl distribution (3D fifth order $\mathrm{MLP}^{l d}$ ) is shown.

\section{EXPERIMENTAL SETUP}

The gaseous hydrogen $\left(\mathrm{GH}_{2}\right)$ and oxygen $\left(\mathrm{GO}_{2}\right)$ single element model rocket combustor (PennState Preburner Combustor) [1, 4] was chosen as the reference case for the following numerical investigations. The use of $\mathrm{GH}_{2}$ and $\mathrm{GO}_{2}$ results from an interest in full-flow staged combustion (FFSC) cycle engines which are relatively simple and offer an increased thrust to weight ratio compared to other closed cycle engines. The experiment was designed to characterize the chamber wall heat transfer. Positions of the temperature and heat flux measurements are given in Fig. 1, where wall temperatures and wall heat flux profiles are plotted (by symbols) along the combustor length. The chamber diameter is $38.1 \mathrm{~mm}$ and its length is $285.75 \mathrm{~mm}$. Two upstream located preburners produce oxidizer-rich and fuel-rich gases. The oxidizer-rich gas is fed to the combustion chamber through the inner tube of the injector which has a diameter of $5.26 \mathrm{~mm}$ and is recessed $0.43 \mathrm{~mm}$ with respect to the combustion chamber face plane. The annular fuel feed has an inner diameter of $6.3 \mathrm{~mm}$ and an outer diameter of $7.49 \mathrm{~mm}$. The oxidizer to fuel ratio is 6.6 and the nominal chamber pressure of this test case is $5.17 \mathrm{MPa}$. However, during operation a chamber pressure of $5.42 \mathrm{MPa}$ was measured. Table I summarizes the operation conditions and geometrical details. 
Table I. Operating conditions and geometrical details of the PennState Preburner Combustor test case [1, 4].

\begin{tabular}{llcc}
\hline & & oxidizer preburner & fuel preburner \\
\hline pressure & $(\mathrm{MPa})$ & 5.85 & 6.50 \\
temperature of products & $(\mathrm{K})$ & 700 & 811 \\
$\mathrm{O}_{2}$ mass flow in products & $(\mathrm{kg} / \mathrm{s})$ & $8.55 \cdot 10^{-2}$ & - \\
$\mathrm{H}_{2}$ mass flow in products & $(\mathrm{kg} / \mathrm{s})$ & - & $1.33 \cdot 10^{-2}$ \\
$\mathrm{H}_{2} \mathrm{O}$ mass flow in products & $(\mathrm{kg} / \mathrm{s})$ & $4.98 \cdot 10^{-3}$ & $1.98 \cdot 10^{-2}$ \\
inner diameter & $(\mathrm{mm})$ & - & 6.30 \\
outer diameter & $(\mathrm{mm})$ & 5.26 & 7.49 \\
\hline & \multicolumn{3}{c}{ main combustion chamber } \\
\hline & \multicolumn{3}{c}{$5.17 / 5.42$} \\
\hline pressure & $(\mathrm{MPa})$ & \multicolumn{2}{c}{28.1} \\
chamber diameter & $(\mathrm{mm})$ & \multicolumn{2}{c}{0.43} \\
length & $(\mathrm{mm})$ & \multicolumn{2}{c}{8.166} \\
recess & $(\mathrm{mm})$ & \multicolumn{3}{c}{} \\
nozzle diameter & $(\mathrm{mm})$ & \multicolumn{3}{c}{} \\
\hline
\end{tabular}

\section{NUMERICAL SETUP}

The numerical setup for the test case follows as much as possible the experimental conditions.

\subsection{Boundary and initial conditions}

For the combustion chamber isothermal walls are chosen with temperatures obtained from a least squares fit of the measured data (see Fig. 1). The injector post tip, the vertical chamber wall and the nozzle walls are treated isothermal with temperatures of $755 \mathrm{~K}$ and $510 \mathrm{~K}$, respectively. At the injector walls adiabatic boundary conditions are set. This is illustrated in Fig. 1. At the inflows precalculated profiles and mass flow boundary conditions are used. The inflow profiles are assumed to be fully developed turbulent pipe flows.

To start the simulation the combustor is initialized with hot water of $3000 \mathrm{~K}$ while the injectors are filled with gases corresponding to their inflow conditions. This procedure enables a fast progress of the simulation since self ignition takes place as soon as the fluids have mixed and heated up due to the hot water. This initialization is numerically stable and no sudden explosions occurs. However, subsequent simulations are started from steady-state solutions which were either interpolated from different grids or taken from previous calculations with differing parameters. 


\subsection{Averaging and time discretization}

Most of the simulations performed in this paper are unsteady calculations. From the time resolved data time-averaged values have to be calculated. To this end the flow field data is averaged over a period of at least three flow-through times, starting after a startup phase of two flow-through times. The startup phase is required to remove influences from the initial conditions. One flow-through time was defined by Tucker et al. [13] to be $8.3 \mathrm{~ms}$ based on the bulk mass flow through the chamber. As mentioned in Sec. 2 a dual time stepping is required for time accurate solutions. All simulations in this paper use a physical time step of $10^{-8}$ seconds. For every physical time step a number of inner iterations has to be performed in pseudo time until a predefined convergence criteria is complied. For the inner iteration this criteria is a decrease in the normalized absolute mean density residual of at least three orders of magnitude. This is usually achieved within 5 to 7 inner iterations.

Time discretization is based on a second order BDF scheme. The second order scheme was found to be numerically more stable than the corresponding 3rd order BDF method which is also available. Differences between both discretizations have been compared and were found to be minor. In comparison with other authors $[15,16]$ the chosen time step is relatively short and the number of inner iterations relatively high. However, these values are required due to the comparatively fine computational grids (especially at the injector and at solid walls) and the high frequency pressure fluctuations close to the injector post.

\subsection{Computational Grids}

All two-dimensional steady-state RANS calculations are performed on a grid with approximately 46,000 volumes. Unfortunately, a significantly coarser grid had to be chosen compared to the unsteady simulations. In agreement with the findings of Lian and Merkle [15] it was not possible to achieve a converged solution on finer grids due to the inherently unsteady behavior of the flow field close to the injector. Thus grid independency could not be achieved for the RANS simulations.

The two-dimensional time accurate simulations (URANS) use a computational grid with approximately 135,000 volumes. Like all meshes used in this paper, it is significantly refined at all 
near wall regions to meet constraints of the low-Reynolds number $k-\omega$ turbulence model $\left(y^{+} \approx 1\right.$ for the first cell centre adjacent to the wall) and to enable an accurate calculation of the wall heat flux. The latter requires a well resolved temperature profile in the viscous sublayer. To check grid convergence of the unsteady simulation an additional finer 2D grid with 540,000 volumes (equally refined in both directions) is used. Results (see sect. 5.1) are compared with those of the reference grid.

An objective comparison between $3 \mathrm{D}$ and $2 \mathrm{D}$ simulations is one of the main ambitions of this paper. Therefore grids with the same axial and radial resolution were chosen. This allows to attribute differences in the solutions reclusively to the third dimension. The 3D URANS grid consists of approximately 16.3 million cells. The number of volumes in the azimuthal direction is 128 . The blocks around the symmetry axis are constructed with a butterfly grid to fit the round geometry into a structured multi-block grid. The time-averaged results from the 3D simulation are spatially averaged in azimuthal direction in order to gain comparable two-dimensional solutions. Due to the high computational effort of the unsteady 3D simulation, the grid could not be refined to check grid convergence as in the 2D case. The computational grid is divided into 3992 blocks and the solution of any block is advanced on a separate CPU of a parallel computing system.

\section{RESULTS AND DISCUSSION}

Simulations are performed for the PennState combustor in order to investigate the differences between steady and time accurate simulations, axisymmetric 2D and 3D simulations, as well as to assess the influence of the order of spatial discretization. To this end results will be presented from the following simulations:

1. two-dimensional RANS using the second order minmod limiter,

2. two-dimensional RANS using the fifth order MLP ${ }^{l d}$ scheme,

3. two-dimensional URANS using the second order minmod limiter,

4. two-dimensional URANS using the second order van Leer limiter, 


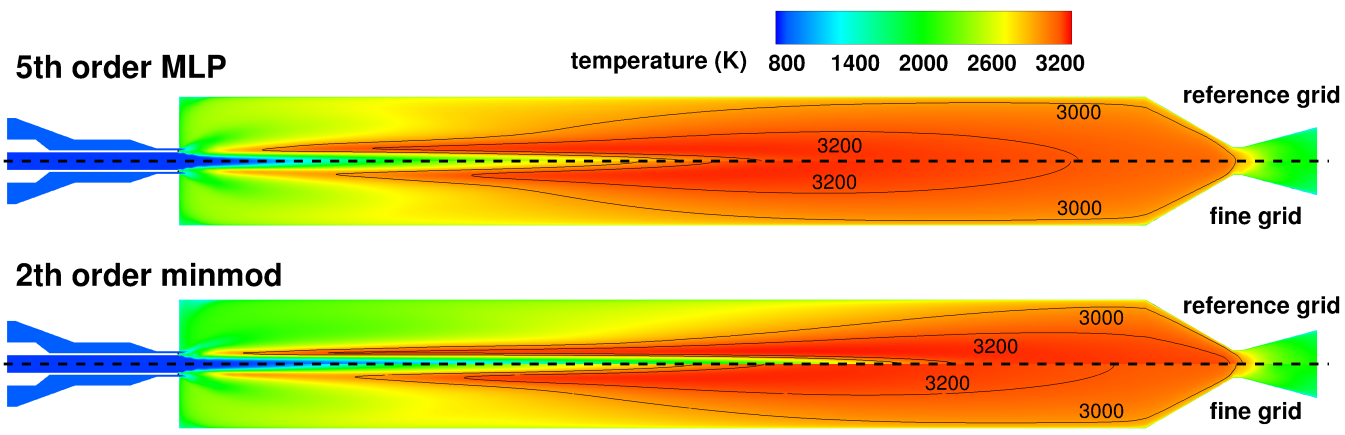

Figure 2. Comparison of averaged temperature contours for 5th order MLP (top) and 2nd order minmod (bottom) discretization. The upper half of any figure is obtained using the reference grid, the lower half is a solution using the refined grid.

5. two-dimensional URANS using the fifth order MLP ${ }^{l d}$ scheme,

6. three-dimensional URANS using the fifth order MLP ${ }^{l d}$ scheme.

Because only wall heat fluxes have been measured in the experiment, it is difficult to assign differences in the numerical results to single fluid phenomena. However, as to gain insight into the different processes flow fields are investigated and compared with results of other research groups.

\subsection{Grid convergence}

For the 2D axisymmetric URANS simulation a grid refinement study is performed. The reference grid for the 2D simulations has been refined by doubling the number of volumes in both coordinate directions (540,000 instead of 135,000 volumes). For this study the second order minmod and the fifth order MLP discretization are used for the inviscid fluxes. Figure 2 shows contour plots of the averaged temperature for the fifth order MLP (top) and the second order minmod (bottom) discretization. Both figures are divided into two parts: the upper half is calculated using the reference grid, the lower half with the refined grid. To visualize the differences in the temperature field two isothermal contour lines at 3000 and $3200 \mathrm{~K}$ are drawn as well. Several key features can be observed from these plots. First, both the type of discretization and the computational grid have large influences on the temperature distribution. Second, the differences between the second order and fifth order discretization decline on the finer grid. Third, on the regular grid the fifth order discretization is in much better agreement with the corresponding solutions on the fine grid, 


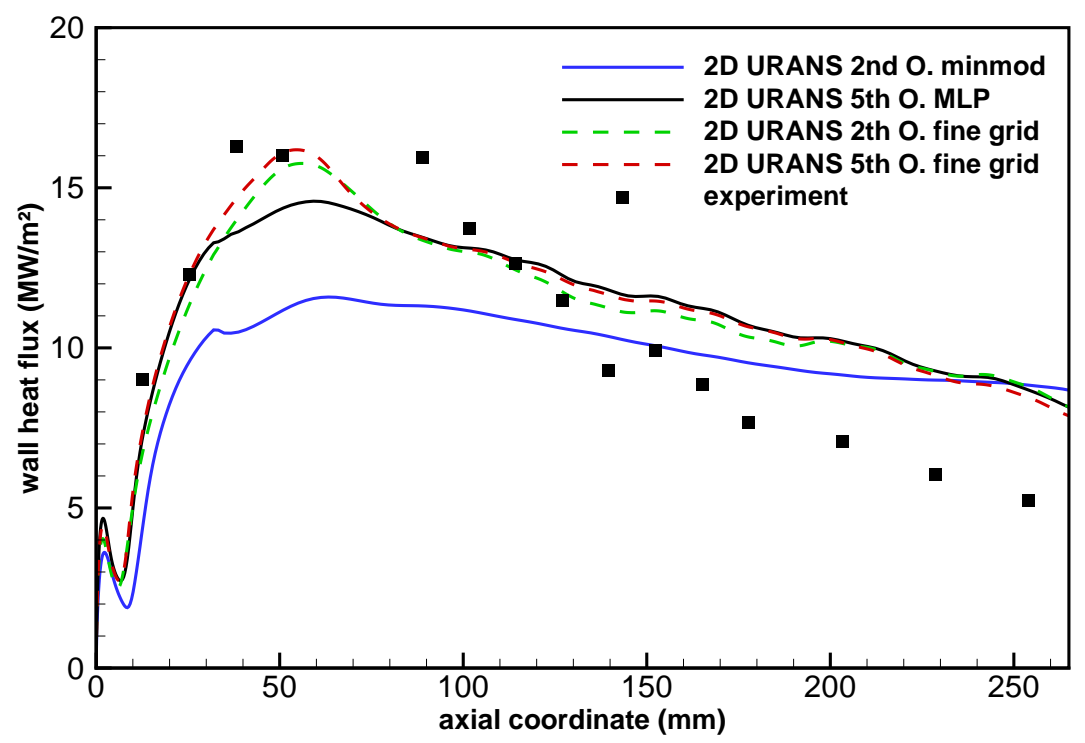

Figure 3. Comparison of the wall heat fluxes for the reference and the refined grid from 2D URANS simulations with 2nd order minmod and 5th order MLP discretization.

compared to the second order discretization. This clearly shows the advantages of using a high order discretization, which has also been demonstrated in [18, 38].

Figure 3 compares the wall heat fluxes of the four simulations (lines) with experimentally measured values (symbols). In case of the fifth order MLP simulation the differences between the fine and the regular grid are minor except for the distinctive peak in the fine grid solution around $x=50 \mathrm{~mm}$. The heat flux of the second order minmod simulation shows large differences to the experiment on the regular grid but comes very close to the fifth order simulation on the fine grid. This shows, that complete grid independency is not achieved on the reference grid neither with the second nor the fifth order discretization. There even are some grid dependencies on the refined grid but at least for the fifth order scheme these are expected to be small. Moreover the fifth order simulation on the regular grid shows much more similarities with the fine grid solution. In summary, the fifth order MLP simulation achieves comparable results to the second order discretization on a grid with doubled number of volumes in any coordinate direction.

For the 2D simulations it would have been possible to use even finer grids, however, not in the 3D case. A doubling of the number of volumes in all coordinate directions (as done for the refined 


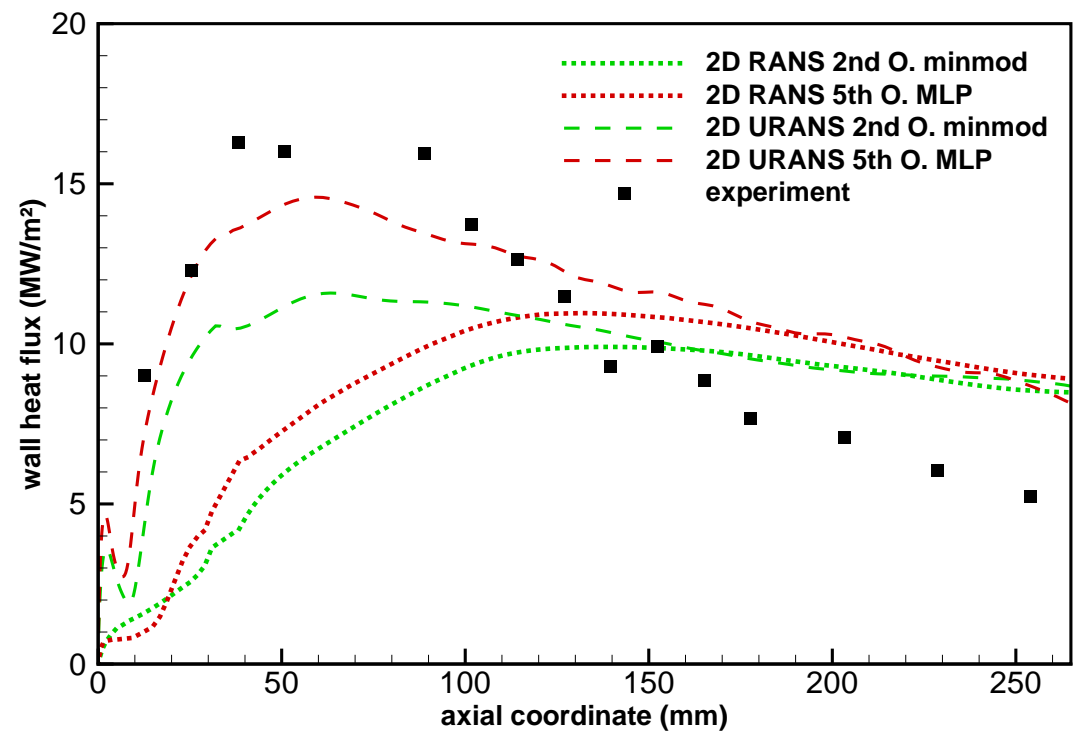

Figure 4. Comparison of the wall heat fluxes from 2D URANS simulations with 2nd order minmod limiter and 5th order MLP (upper two lines) discretization. In addition two RANS results are given (using the minmod limiter and 5th order MLP).

grid in 2D) would result in 130 million volumes in 3D, what, with finite-rate chemistry, exceeds our available computational resources. Note, that in literature most LES for this test case (with exception of the SANDIA simulation [13]) use much coarser grids than the present URANS. Moreover this is the only study where a 5th order spatial discretization is used. To ensure the comparability between two- and three-dimensional simulations, grids with the same axial and radial resolution are essential. This allows to attribute differences in the solutions reclusively to the third spatial dimension. For this reason the reference grid is chosen for the $2 \mathrm{D}$ and (its extended version) $3 \mathrm{D}$ simulations. The general statements of the following 2D/3D comparison as well as of the other studies performed most likely are not influenced by the still existing grid dependency.

\subsection{Axisymmetric two-dimensional RANS}

As can be observed from Fig. 4 both RANS simulations (with second order minmod limiter and the 5th order MLP ${ }^{l d}$ discretization) are not able to reproduce the experimentally measured heat fluxes. Reason is a combustion zone which is extremely thin (see Fig. 6) and extends up to the nozzle. The highest $\mathrm{OH}$ concentrations are reached shortly upstream of the nozzle throat (see Fig. 7). As a 
consequence a very large recirculation zone is obtained (see streamlines in Fig. 7) which basically is responsible for the erroneous heat fluxes. The Wilcox $k-\omega$ turbulence model has been used in its latest version [27] for these simulations. As mentioned above these steady-state RANS have been possible on a coarse grid only, confirming the findings of Lian and Merkle [15]. No attempts have been made to improve the RANS results by changing parameters or coefficients (e.g. turbulent Prandtl and Schmidt number). For scientific simulations the unsteady effects close to the injector were found to be important requiring an unsteady simulation and a significantly finer grid.

\subsection{High order discretization}

As observed in previous studies $[17,35,38]$ the quality of numerical results can be significantly improved by changing to higher order spatial discretizations. MLP ${ }^{l d}$ offers the possibility of a stable high order scheme with good shock capturing capabilities. The latter may be required in the nozzle in case of an unmatched pressure. The wall heat fluxes plotted in Fig. 4 confirm these statements. Results of the 5th order URANS scheme agree much better with the experimental data than corresponding simulations with the second order minmod limiter. The latter is known to be very dissipative. A second order URANS simulation with van Leer limiter (not shown in this figure) is somewhat better but it is still much closer to the minmod heat flux than to the fifth order result. Taking into account that the additional numerical cost for the high order scheme is extremely low (less than $10 \%$ ) and that the stability of $\mathrm{MLP}^{l d}$ is very good the advantages become evident.

\subsection{Three-dimensional URANS simulation}

All simulated heat fluxes of Fig. 4 have in common that downstream of the maximum (at $x>$ $100 \mathrm{~mm}$ ) the numerical values are significantly to high. In this region the full three-dimensional simulation shown in Fig. 5 is much closer to the experimental results. In particular the downward slope of the measured data is well captured. For comparison this figure also includes the $2 \mathrm{D}$ axisymmetric 5th order URANS results from the last figure. Moreover, wall heat fluxes from two other reserach groups, SANDIA National Laboratory (SNL) and Georgia Tech (GT) [13], 


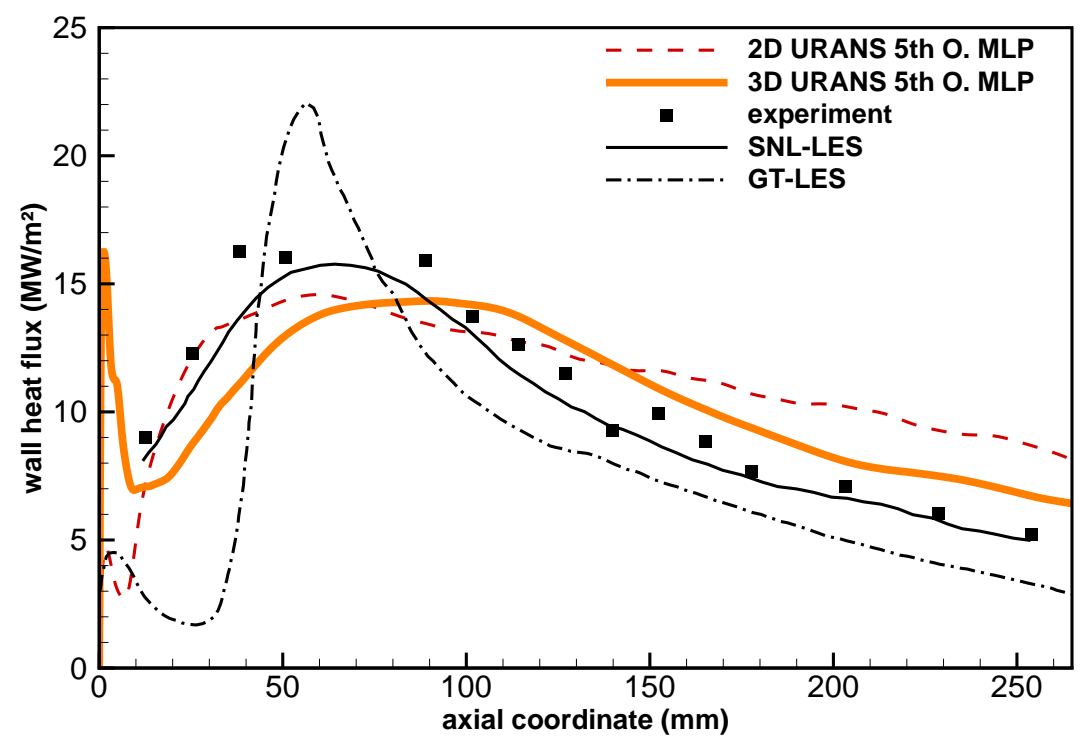

Figure 5. Comparison of the wall heat fluxes of a 2D axisymmetric and a full 3D URANS simulation with 5th order $\mathrm{MLP}^{l d}$ discretization. In addition LES results from the SANDIA National Laboratory (SNL) and Georgia Tech (GT) [13] are given.

are given. As may be seen from this figure the integral heat release of the present 3D simulation in the instrumented domain agrees very well with the experiment. A nearly constant offset of approximately $20 \mathrm{~mm}$ in downstream direction and a slight underprediction of the maximum heat flux can be observed. Another positive point is that the structure of the heat flux profile agrees much better with the experiment than for the $2 \mathrm{D}$ simulation. Thus it can be stated, that with increasing complexity of the simulation the wall heat fluxes agree better with the measured data. As will be shown later the 3D discretization has a tremendous effect on mixing and combustion. While the present 3D URANS heat fluxes are better than those of the LES from GT (using 3.2 million volumes), the SNL profile (using 255 million volumes) agrees nearly perfectly with the experiment.

\subsection{Flow field and discussion}

Figures 6 and 7 show temperature and $\mathrm{OH}$ distributions of all investigated test cases. Huge differences become evident stemming from the type of simulation as well as from discretization. The figures show a clear trend from top to bottom. In this order the complexity of the simulation (concerning the quality of spatial and temporal discretization and of the computational grid) is 


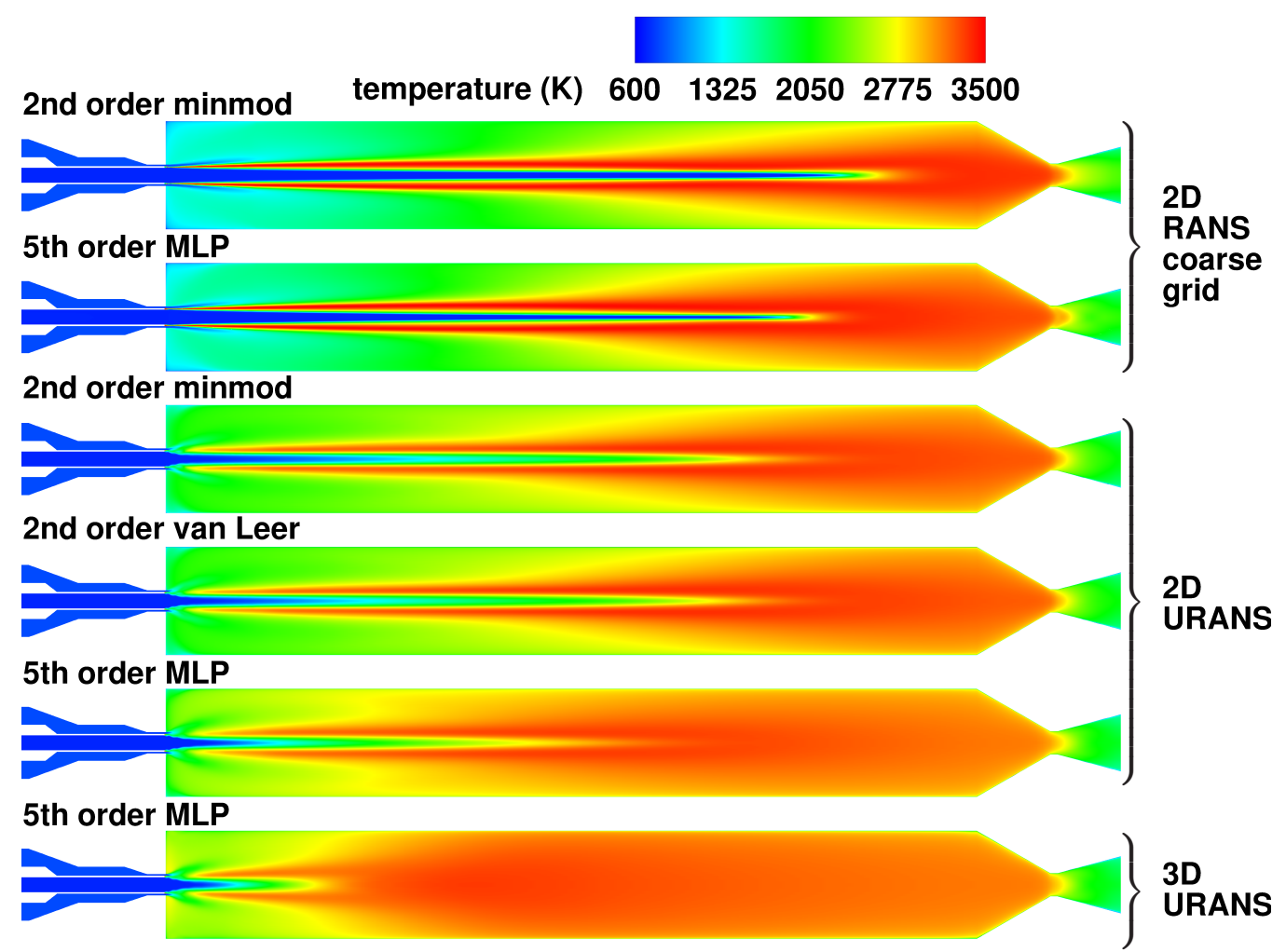

Figure 6. Comparison of time averaged temperature distributions for simulations with increasing complexity from top to bottom.

increased. In the 2D axisymmetric RANS simulations combustion and heat release take place in a long narrow tube which hardly spreads in radial direction. Just ahead of the nozzle throat the OH distributions get broader. The temperature plots for the RANS simulations clearly show a cold oxygen jet which is visible over 70-80 \% of the combustor length. Such a long stable oxygen jet probably will not exist in reality. The change from RANS to URANS intensifies the turbulent mixing due to unsteadiness which in the latter case is covered by the simulation. As a consequence the shear layer and the flame get broader, peak temperatures decrease, and the combustion zone is moving upstream. These effects are enhanced with the reduction of numerical dissipation, achieved by changing from the most dissipative second order minmod to the less dissipative van Leer limiter and further to the 5 th order MLP ${ }^{l d}$ scheme. This mere increase in order of discretization shows a tremendous impact on the results. Due to the upstream movement of the flame, the size of the 


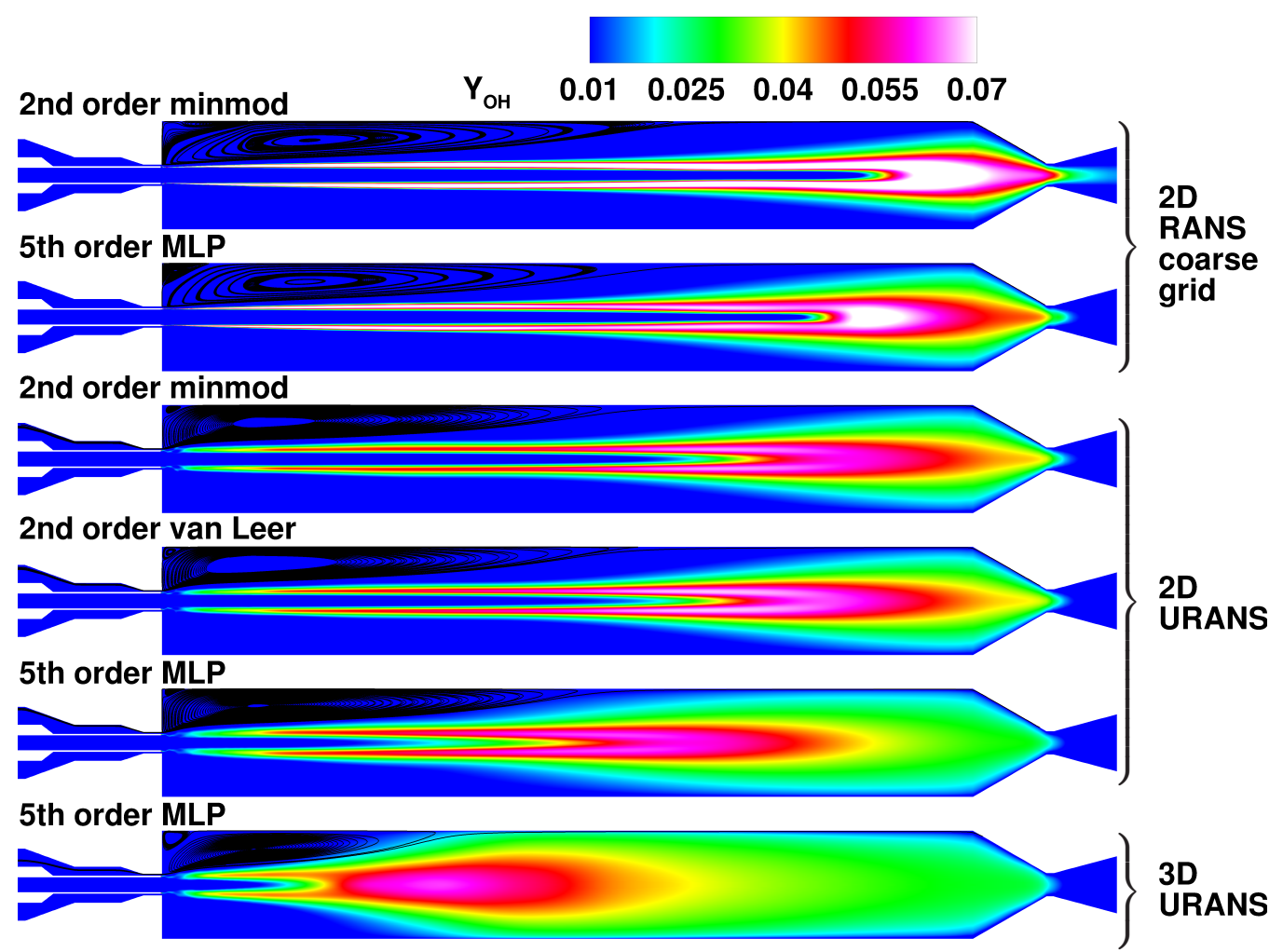

Figure 7. Comparison of time averaged $\mathrm{OH}$ mass fraction distributions and recirculation zones for simulations with increasing complexity from top to bottom.

recirculation zone is reduced (see Fig. 7). The expansion of the gas associated with an increasingly extensive combustion zone causes this reduction.

The third type of simulation (after axisymmetric RANS and URANS), the 3D URANS with 5th order MLP discretization, causes a further change, which is most prominent in the $\mathrm{OH}$ distribution. The flame dramatically reduces in size and moves still more upstream (see the two most below figures of Fig. 7). This is in agreement with most LES, for example those of Georgia Tech [13]. Combustion is much more intense now and completely limited to the first part of the combustor. This additional change is credited to the removal of the axisymmetric constraint at the center line [15] and at both sides. Due to the upstream movement and the more compact flame the wall temperature decreases in the second half of the combustor. This induces the decreased heat fluxes at $x>100$ $\mathrm{mm}$ in case of the 3D simulation (see Fig. 5). 

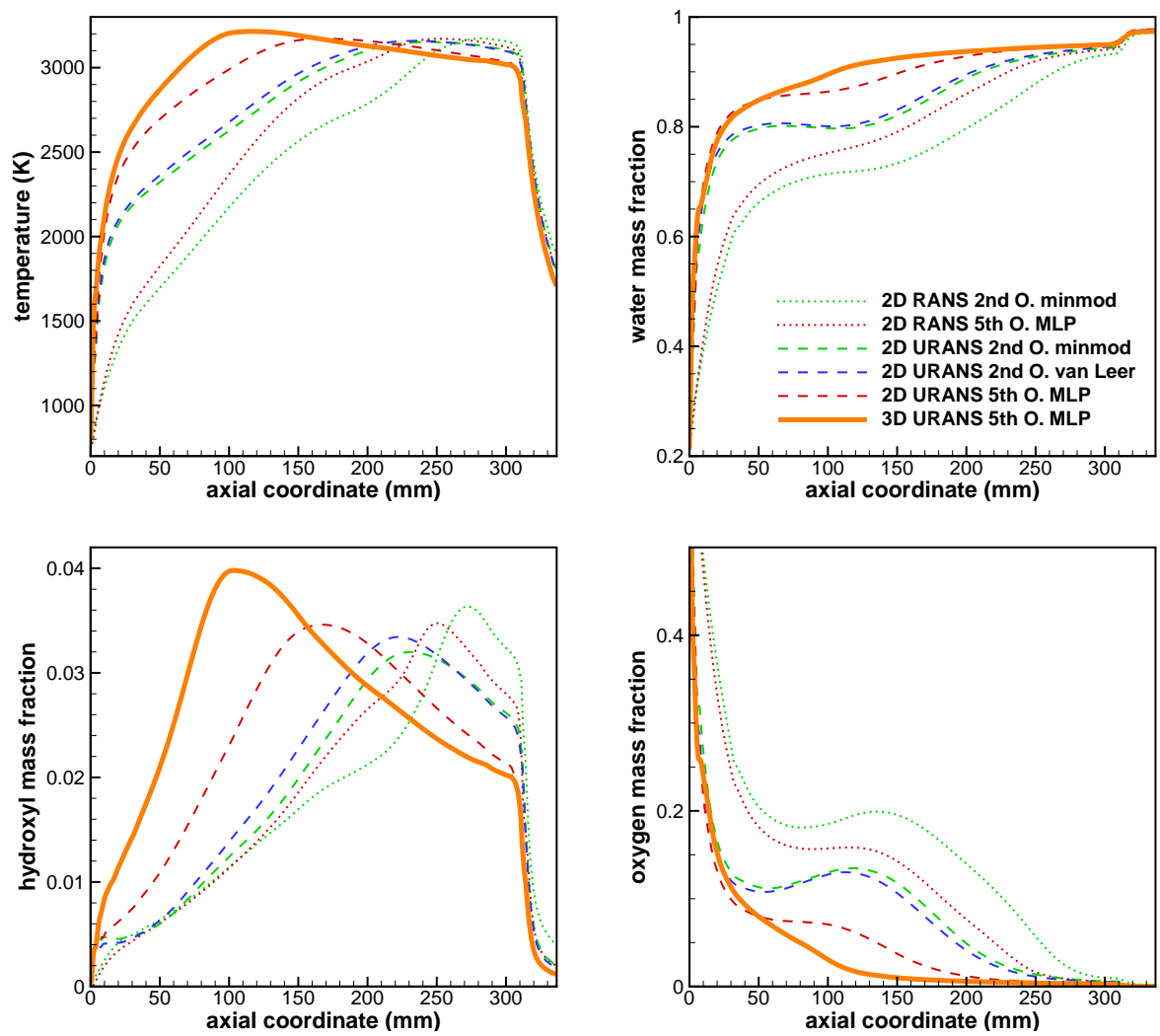

Figure 8. Mass flux cross section averaged values of temperature (top left), water (top right), hydroxyl (bottom left), and oxygen (bottom right) mass fractions along the combustor length for the different simulations.

To get some more insight into mixing and combustion, mass flux averaged values are calculated for every channel cross section, which are functions of the combustor length only. Figure 8 shows averaged values of temperature, water, hydroxyl, and oxygen mass fractions. These profiles clarify the statements given above. The change from 2D RANS to 2D URANS and 3D URANS causes an upstream movement of the flame which is visible in the temperature, water, and oxygen profiles. These cross section averaged values also visualze the tremendous effects stemming from the change in discretization. The temperature profiles show how the 3D URANS simulation reaches its maximum temperature (at $x \approx 100 \mathrm{~mm}$ ) much faster than the other simulations. Combustion is basically completed in the 3D URANS simulation approximately $140 \mathrm{~mm}$ downstream of the injector, as can be seen from the oxygen profile. However, due to the high temperatures, $\mathrm{OH}$ is still present at significant concentrations until the flow is accelerated in the nozzle. 
Table II. Combustion chamber pressures and total heat losses through the combustor wall (without nozzle) for different URANS simulations.

\begin{tabular}{|c|c|c|}
\hline & pressure $(\mathrm{MPa})$ & total heat loss (MW) \\
\hline 2D 2nd order minmod & 5.274 & 0.326 \\
\hline 2D 2nd order van Leer & 5.086 & 0.333 \\
\hline 2D 5th order MLP ML $^{l d}$ & 4.510 & 0.375 \\
\hline 3D 5th order MLP $\mathrm{ML}^{l d}$ & 4.738 & 0.348 \\
\hline
\end{tabular}

\subsection{Pressure level in the combustor}

In the present study a compressible flow solver is used which allows a complete combustor simulation including the nozzle. Thus the combustor pressure is obtained from the simulation which is defined by the inflow conditions, wall heat losses, combustion efficiency, and nozzle throat cross section. In Tab. II the mean pressures in the combustion chamber and the total energy losses by wall heat fluxes (up to $x=285 \mathrm{~mm}$ and excluding the convergent nozzle part) are given. The RANS simulations are not included in this table because they have have been performed on a much coarser grid. The measured pressure of $5.42 \mathrm{MPa}$ is not reached by any of the presented calculations. This is the case for numerical simulations of other authors, too [15], and some possible reasons are given in [14]. The different types of discretization cause significant pressure differences similar to the observed deviations in other variables. However, there is a clear relationship between pressure and wall heat loss. With increasing wall heat loss the pressure decreases. This indicates that remaining differences in heat release up to the nozzle are minor and do not (at least not significantly) change the pressure level.

\subsection{Comparison with LES of other research groups}

In this section profiles from the 5th order 3D URANS simulation are compared with second order LES from SNL and third order upwind biased TVD simulations from GT [13]. To the knowledge of the authors, the LES from SNL is the most elaborate simulation of the PennState Preburner Combustor which shows excellent wall heat flux results. For two axial positions $(x=50 \mathrm{~mm}$ and $150 \mathrm{~mm}$ ) the temperature, hydrogen, and $\mathrm{OH}$ mass fraction profiles (from top to bottom) are plotted in Fig. 9 in radial direction. As may be seen from these figures the present high order URANS 

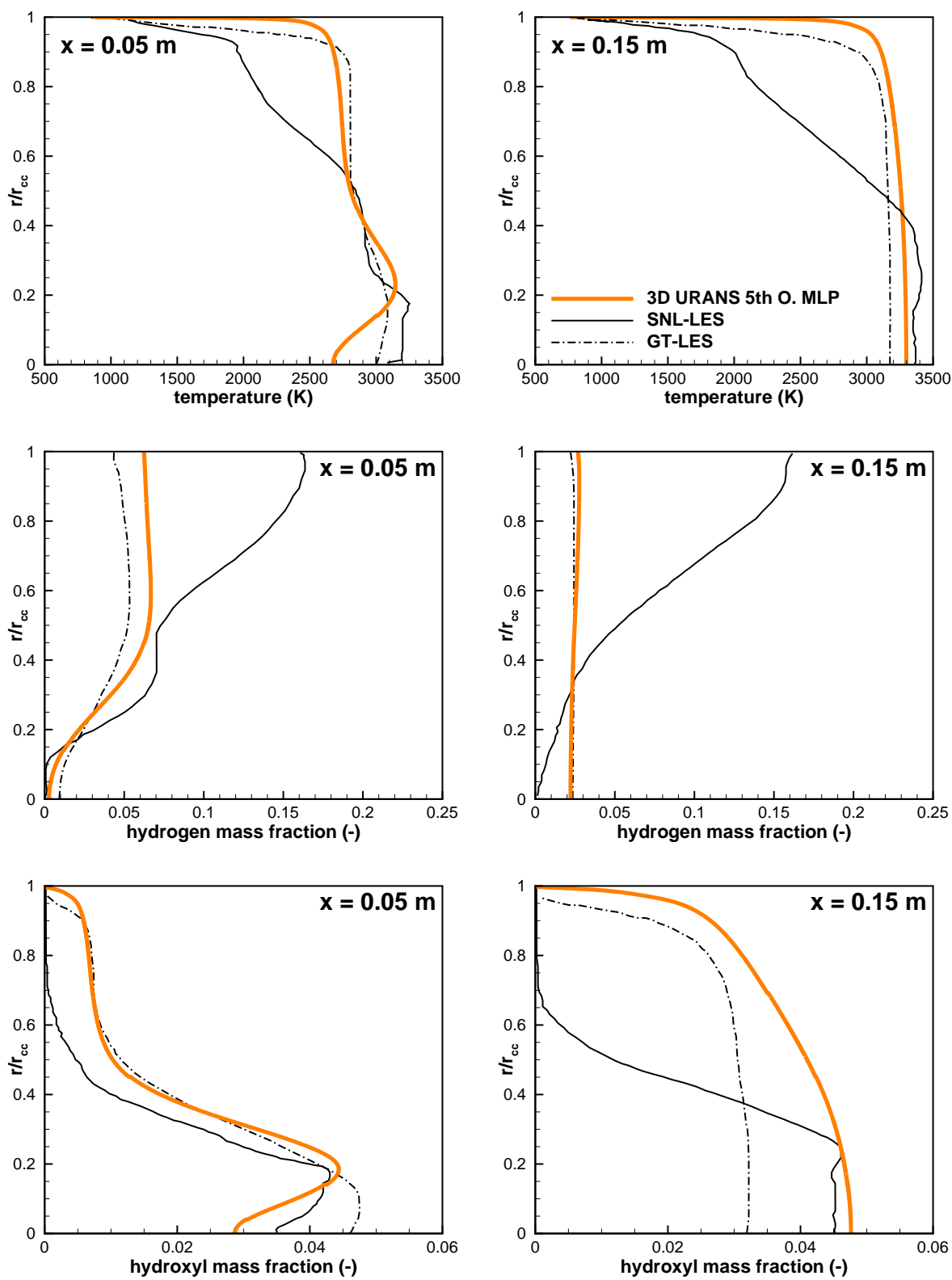

Figure 9. Radial profiles (left at $\mathrm{x}=0.05 \mathrm{~m}$, right at $\mathrm{x}=0.15 \mathrm{~m}$ ) for temperature (top), hydrogen (middle) and $\mathrm{OH}$ (bottom) distributions in comparison to two 3D-LES from literature [13].

simulation and the GT LES agree very well. In the temperature profiles the greatest differences occur in the boundary layer, which is insufficiently resolved in case of the GT simulation [13]. This may be the reason why the present URANS achieves better results for the wall heat fluxes. Larger deviations between the present results and those from GT appear in the $\mathrm{OH}$ profile at $x=150 \mathrm{~mm}$ 
only. While the structure of the profiles remain similar the values from GT are lower. This may be caused by the different reaction mechanisms or the modeling of turbulence-chemistry interaction.

The LES from SNL shows a completely different behavior compared to the present and all other published results, known to the authors. It shows very high hydrogen concentrations along the walls which over the complete combustor length hardly mix with the interior flow (see Fig. 9). The high hydrogen concentrations towards the wall are associated with a unique structure of recirculation zones [13]. These are also responsible for significantly reduced temperatures in the upper and lower quarters of the combustor. Thus tremendous differences to the present simulation exist in the flow field despite the similar wall heat flux profiles in both cases. The reasons for this are still open. However, it clearly indicates that experimental wall heat fluxes alone are insufficient for a meaningful validation.

\subsection{Dynamic flow features}

Transient data have been recorded for selected variables at six monitoring positions for the URANS simulations with 5th order MLP discretization. Three of these positions are located near the injector face, two further downstream at the combustion chamber wall, and one in the convergent part of the nozzle. In case of the three-dimensional simulation four points are evenly distributed in azimuthal direction at each radial position. These data are analyzed by Fast Fourier Transformation (FFT). The obtained frequency spectra at all monitoring points agree quantitatively for all recorded variables (pressure, velocity, temperature, and wall heat flux) independently of the numerical approach. Figure 10 shows representative spectras of axial velocity (upper figure) and pressure (lower figure). The upper figure is obtained at $x=-2 \mathrm{~mm}$ and $y=3.45 \mathrm{~mm}$ (slightly upstream of the injector face inside the annular fuel tube) and the lower figure at $x=310 \mathrm{~mm}$ and $y=2 \mathrm{~mm}$ (in the convergent nozzle part). Thus the two monitoring points are at opposite sides of the combustor. Two distinct frequencies as well as overtones are clearly visible in all investigated spectra (also those not shown in the paper). The lower frequency denoted "A" has a value of approximately $2410 \mathrm{~Hz}$. This is in excellent agreement with the findings of [16] and corresponds to the fundamental longitudinal 

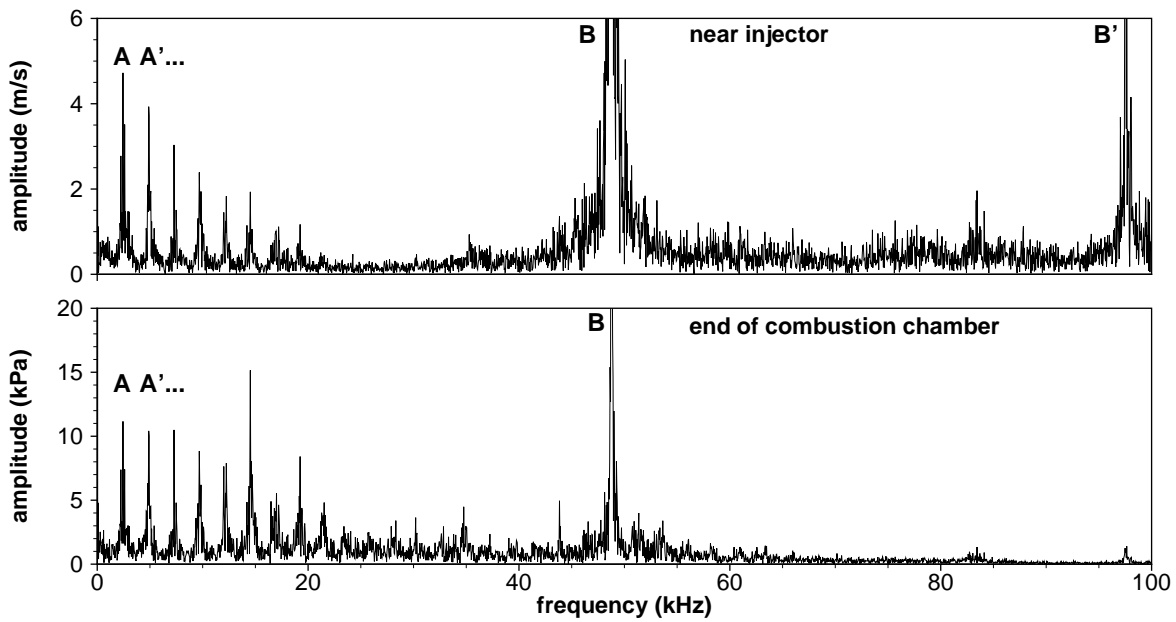

Figure 10. Frequency spectra of axial velocity near the injector (upper figure) and pressure in the convergent nozzle part (lower figure).

acoustic mode of the chamber $f_{L}=\bar{c} / 2 L$, where $\bar{c}$ is the average speed of sound in the combustion chamber and $L$ is its length. The amplitudes of these oscillations are comparatively low, typically below $1 \%$ of the average value. In [16] a frequency offset between pressure and wall heat flux oscillations is observed. This is not the case in the present simulation where the frequency peaks coincide (not shown in the paper).

The high frequency oscillation at $48.8 \mathrm{kHz}$ (denoted "B") results from dynamic instabilities in the shear layer of the coaxial jet. According to [39] the Strouhal number of the primary vortices in a coaxial jet configuration can be calculated by

$$
S t=\frac{0.6 f D_{i}}{0.6\left(\bar{U}_{o}-\bar{U}_{i}\right)+\bar{U}_{i}},
$$

where $D$ are diameters, $\bar{U}$ is the bulk velocity, and the indices $i$ and $o$ denote the inner (oxidizer) and outer (fuel) jet, respectively. For the investigated configuration and a frequency of $48.8 \mathrm{kHz}$ a Strouhal number of $S t=0.3$ is obtained. According to [39] vortices with Strouhal numbers between 0.3 and 0.5 are preferred modes and experience continuous growth. This is in accordance with the 


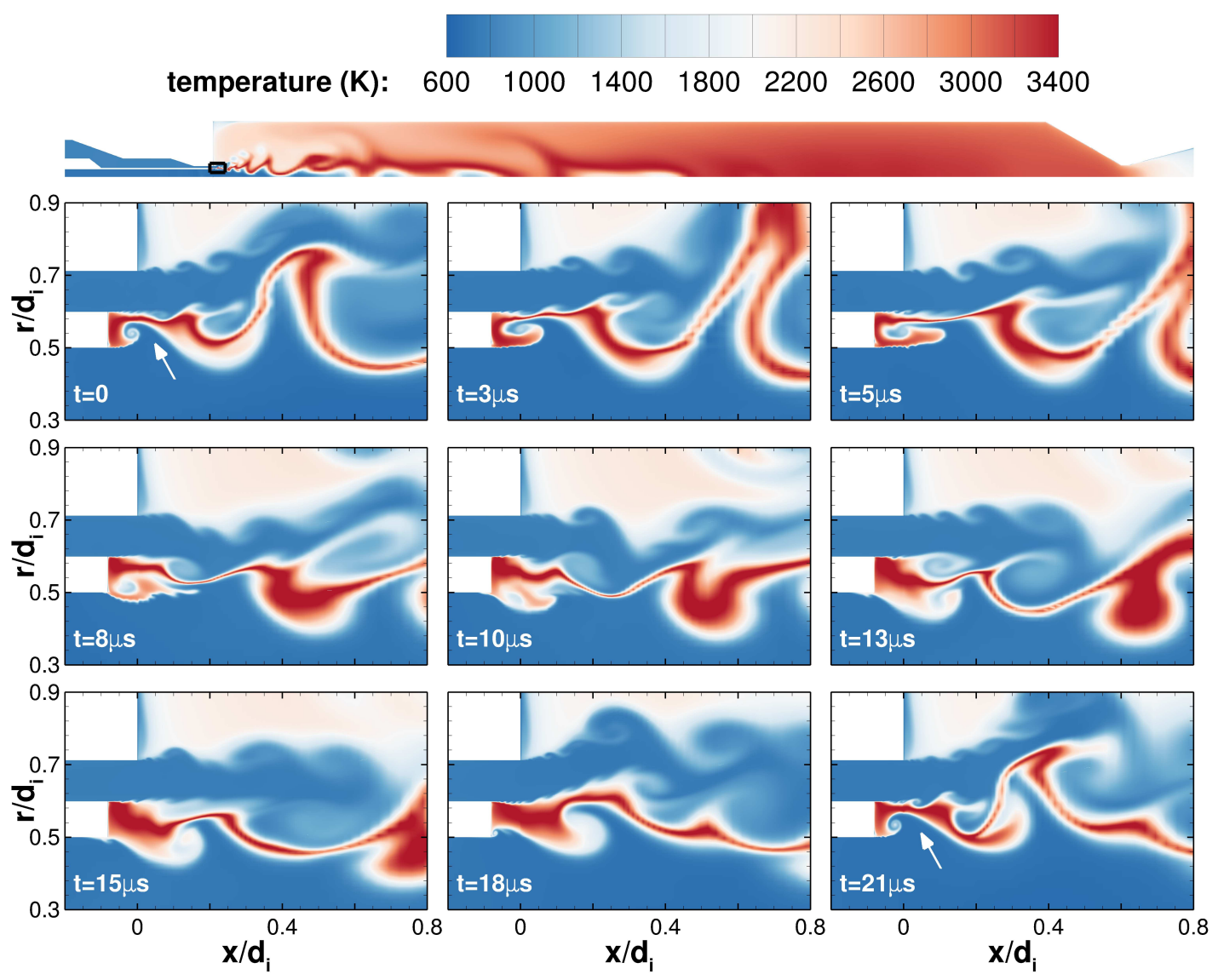

Figure 11. Series of instantaneous snapshots of temperature contours close to the injector. The flow fields advance in time from left to right and from top to bottom (from $t=0$ up to $t=21 \mu \mathrm{s}$ ).

pressure spectra, where the highest amplitudes observed are close to the injector with fluctuations of up to $8 \%$ of the average value.

To provide further evidence that the $48.8 \mathrm{kHz}$ mode is connected to the production of vortices in the inner shear layer of the coaxial jet, Fig. 11 shows instantaneous snapshots of temperature in the near injector region at nine instances in time. The time $t_{0}=0$ is chosen arbitrarily and the sequence is organized from left to right and top to bottom.

There is an obvious similarity between the temperature contours at $t=0$ and $t=21 \mu \mathrm{s}$. At both times a counterclockwise rotating vortex is formed directly behind the oxidizer post tip, which suggests a periodic behavior with a period of approximately $21 \mu \mathrm{s}$. This vortex transports low 


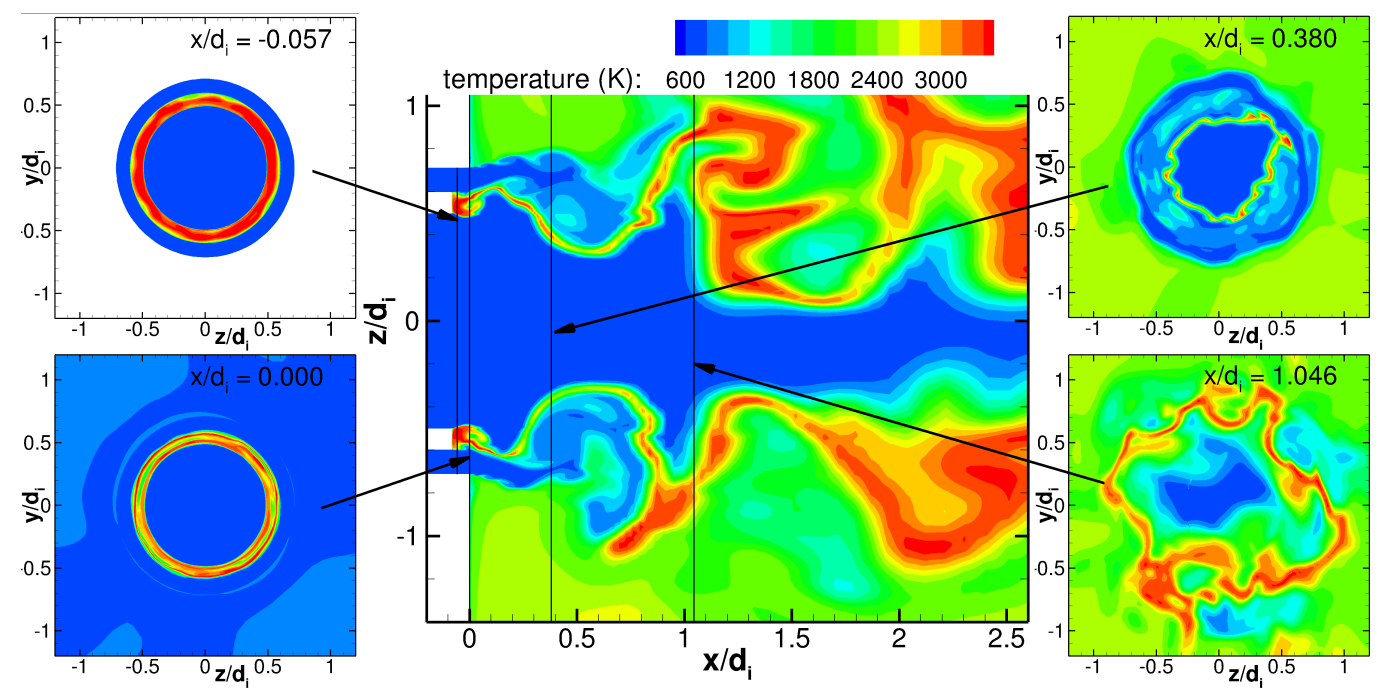

Figure 12. Instantaneous snapshots of temperature contours of the 3D simulation in different planes close to the injector. In the middle the $\mathrm{y}=0$ plane is shown. Left and right are different $\mathrm{x}$ planes.

temperature oxidizer rich gases into the combustion zone and at $t=18 \mu$ s has moved downstream with the result that a new vortex may be formed at the oxidizer post tip. Based on the identified period of approximately $21 \mu \mathrm{s}$, the frequency of the vortex formation is $47.6 \mathrm{kHz}$ which is close to the $48.8 \mathrm{kHz}$ observed in the spectra.

The series of figures show how the different vortices are formed and stretched in time with the hot combustion zone in between. There is a strong fluctuation of the flame in radial direction. The downstream movement of the corresponding vortices causes the strong differences between the URANS and RANS simulations as observed before. These differences are not limited to the near injector region. It is also important to note that the flame is anchored at the oxidizer post tip at all times. This is in accordance with general experimental findings for $\mathrm{H}_{2} / \mathrm{O}_{2}$ rocket combustors [40]. The described periodic processes cause a corrugated flame shape and an alternating contraction and expansion of the central oxygen jet directly at the oxidizer post tip. This is associated with the strong pressure oscillations observed. From Fig. 12 it becomes apparent that the initial vortex shedding occurs in a rotationally symmetric manner (plots on the left). The flow field becomes asymmetric 
quite fast (plots, on the right, see also Fig. 1). This punctuates the necessity of a fully threedimensional simulation. The high-frequency fluctuations associated with the vortex shedding are detectable in the entire chamber. Moreover, these fluctuations have no phase shift in the azimuthal direction at the injector or anywhere in the chamber.

\section{CONCLUSIONS}

A detailed numerical study of the PennState Preburner Combustor is presented. Because the same code was used for all simulations, differences in the results may be directly assigned to the parameter changed. A new 5th order upwind biased discretization technique (MLP ${ }^{l d}$ ) has been used for the first time to simulate a complex 3D combustor with finite-rate chemistry. With this technique, the numerical results could be improved significantly at low additional computational cost (less than $10 \%)$. Moreover, the stability of the 5th order discretization was comparable to those of the second order van Leer limiter. As one might have expected, the 3D URANS with 5th order discretization achieved best results. This may be seen in the wall heat fluxes but also by comparison with the LES from GT. What was not to be expected was the tremendous impact of the investigated discretization techniques, both on the heat flux but also on combustion and flow field. A high order discretization helps to reduce the high computational cost. By a time resolved analysis of the near injector region reasons for the formation of high frequency pressure fluctuations could be given. Nevertheless there still are a number of open questions which arise due to differences to the LES from SNL which have to be clarified in the future. This point also stresses the importance of experiments which besides wall heat fluxes provide additional data to assist the validation of numerical codes.

\section{ACKNOWLEDGEMENTS}

We wish to thank the German Research Foundation (Deutsche Forschungsgemeinschaft - DFG) for financial support in the framework of the Sonderforschungsbereich/Transregio 40. Computational resources 
have been provided by the High Performance Computing Center Stuttgart (HLRS). The third author also wishes to thank the DLR project ProTau.

\section{REFERENCES}

1. Marshall WM, Pal S, Woodward RD, Santoro RJ. Benchmark wall heat flux data for a $\mathrm{GO}_{2} / \mathrm{GH}_{2}$ single element combustor. 41 ${ }^{\text {st }}$ AIAA/ASME/SAE/ASEE Joint Propulsion Conference \& Exhibit, AIAA 2005-3572, Tucson, Arizona, 2005.

2. Grisch F, Bouchardy P, Vingert L, Clauss W, Oschwald M, Stel'mack OM, Smirnov VV. CARS Measurements at High Pressure in Cryogenic LOX/GH2 Jet Flames, Progress in Astronautics and Aeronautics, vol. 200, chap. 10. American Institute of Aeronautics and Astronautics, 2004; 369-404.

3. Locke JM, Pal S, Woodward RD, Santoro RJ. Toward time-resolved measurements in a gaseous hydrogen/oxygen rocket. $45^{\text {th }}$ AIAA/ASME/SAE/ASEE Joint Propulsion Conference \& Exhibit, AIAA 2009-5395, 2009.

4. Pal S, Marshall W, Woodward R, Santoro R. Wall heat flux measurements for a uni-element $\mathrm{GO}_{2} / \mathrm{GH}_{2}$ shear coaxial injector. Third International Workshop on Rocket Combustion Modeling, 2006.

5. Locke JM, Pal S, Woodward RD. Chamber wall heat flux measurements for a $\mathrm{LOX} / \mathrm{CH}_{4}$ uni-element rocket. $43^{\text {rd }}$ AIAA/ASME/SAE/ASEE Joint Propulsion Conference \& Exhibit, AIAA 2007-5547, 2007.

6. TNF workshop; URL http://www. sandia.gov/TNF/abstract.html.

7. Tucker PK, Menon S, Merkle CL, Oefelein JC, Yang V. An approach to improved credibility of CFD simulations for rocket injector design. 43 ${ }^{\text {rd }}$ AIAA/ASME/SAE/ASEE Joint Propulsion Conference \& Exhibit, AIAA 2007-5572, 2007.

8. Ivancic B, Riedmann H, Frey M, Knab O, Karl S, Hannemann K. Investigation of different modeling approaches for CFD simulation of high pressure rocket combustors. $5^{\text {th }}$ European Conference for Aeronautics and Space Sciences (EUCASS), 2013.

9. Oefelein JC, Yang V. Modeling high-pressure mixing and combustion processes in liquid rocket engines. Journal of Propulsion and Power 1998; 14(5):843-857, doi:10.2514/2.5349.

10. Yang V. Modeling of supercritical vaporization, mixing, and combustion processes in liquid-fueled propulsion systems. Proceedings of the Combustion Institute 2000; 28:925-942, doi:10.1016/S0082-0784(00)80299-4.

11. Cheng GC, Farmer R. Real fluid modeling of multiphase flows in liquid rocket engine combustors. Journal of Propulsion and Power 2006; 22(6):1373-1381, doi:10.2514/1.17272.

12. Masquelet M, Menon S, Jin Y, Friedrich R. Simulation of unsteady combustion in a $\mathrm{LOX}-\mathrm{GH}_{2}$ fueled rocket engine. Aerospace Science and Technology 2009; 13:466-474, doi:10.1016/j.ast.2009.07.005.

13. Tucker PK, Menon S, Merkle CL, Oefelein JC, Yang V. Validation of high-fidelity cfd simulations for rocket injector design. $44^{\text {th }}$ AIAA/ASME/SAE/ASEE Joint Propulsion Conference \& Exhibit, AIAA 2008-5226, 2008. 
14. Riedmann H, Kniesner B, Frey M, Munz CD. Modeling of combustion and flow in a single element $\mathrm{GH}_{2} / \mathrm{GO}_{2}$ combustor. CEAS Space Journal 2014; 6(1):47-59, doi:10.1007/s12567-013-0056-3.

15. Lian C, Merkle CL. Contrast between steady and time-averaged unsteady combustion simulations. Computers \& Fluids 2011; 44(1):328-338, doi:10.1016/j.compfluid.2011.01.032.

16. Masquelet M, Menon S. Large-eddy simulation of flame-turbulence interactions in a shear coaxial injector. Journal of Propulsion and Power 2010; 26(5):924-935, doi:10.2514/1.48023.

17. Kim KH, Kim C. Accurate, efficient and monotonic numerical methods for multi-dimensional compressible flows: Part II: Multi-dimensional limiting process. Journal of Computational Physics 2005; 208(2):570-615, doi: 10.1016/j.jcp.2005.02.022.

18. Gerlinger P. Multi-dimensional limiting for high-order schemes including turbulence and combustion. Journal of Computational Physics 2012; 231(5):2199-2228, doi:10.1016/j.jcp.2011.10.024.

19. Gerlinger P, Stoll P, Brüggemann D. An implicit multigrid method for the simulation of chemically reacting flows. Journal of Computational Physics 1998; 146:pp. 322-345, doi:10.1006/jcph.1998.6061.

20. Gerlinger P, Möbus H, Brüggemann D. An implicit multigrid method for turbulent combustion. Journal of Computational Physics 2001; 167(2):247-276, doi:10.1006/jcph.2000.6671.

21. Gerlinger P. Investigation of an assumed pdf approach for finite-rate chemistry. Combustion Science and Technology 2003; 175(5):841-872, doi:10.1080/00102200302410.

22. Gerlinger P, Stoll P, Kindler M, Schneider F, Aigner M. Numerical investigation of mixing and combustion enhancement in supersonic combustors by strut induced streamwise vorticity. Aerospace Science and Technology Mar 2008; 12(2):159-168, doi:10.1016/j.ast.2007.04.003.

23. Gerlinger P, Nold K, Aigner M. Influence of reaction mechanisms, grid spacing, and inflow conditions on the numerical simulation of lifted supersonic flames. International Journal for Numerical Methods in Fluids 2010; 62(12):1357-1380, doi:10.1002/fld.2076.

24. Gerlinger P, Brüggemann D. Multigrid convergence acceleration for turbulent supersonic flows. International Journal for Numerical Methods in Fluids 1997; 24:pp. 1019-1035, doi:10.1002/(SICI)1097-0363(19970530)24: 10〈1019::AID-FLD528 $>3.0 . C O ; 2-O$.

25. Gerlinger P, Brüggemann D. An implicit multigrid scheme for the compressible Navier-Stokes equations with low-Reynolds-number turbulence closure. Journal of Fluids Engineering 1998; 120:pp. 257-262, doi:10.1115/1. 2820642.

26. Gerlinger P, Noll B, Aigner M. Assumed pdf modeling and pdf structure investigation using finite-rate chemistry. Progress in Computational Fluid Dynamics, an International Journal 2005; 5(6):334-344.

27. Wilcox DC. Formulation of the $k$-w turbulence model revisited. AIAA Journal 2008; 46(11):2823-2838, doi: $10.2514 / 1.36541$.

28. Jameson A, Yoon S. Lower-upper implicit schemes with multiple grids for the Euler equations. AIAA Journal 1987; 25(7):929-935, doi:10.2514/3.9724. 
29. Shuen JS. Upwind differencing and LU factorization for chemical non-equilibrium navier-stokes equations. Journal of Computational Physics 1992; 99(2):233-250, doi:10.1016/0021-9991(92)90205-D.

30. Ó Conaire M, Curran HJ, Simmie JM, Pitz WJ, Westbrook CK. A comprehensive modeling study of hydrogen oxidation. International Journal of Chemical Kinetics 2004; 36(11):603-622, doi:10.1002/kin.20036.

31. Keller R, Lempke M, Simsont YH, Gerlinger P, Aigner M. Parallelization and performance analysis of an implicit compressible combustion code for aerospace applications. High Performance Computing in Science and Engineering'14. Springer, 2015; to be published.

32. Venkateswaran S, Merkle CL. Dual time stepping and preconditioning for unsteady computations. $33^{\text {th }}$ Aerospace Sciences Meeting \& Exhibit, Reno, Nevada, AIAA 1995-0078, 1995.

33. Liou MS. A sequel to AUSM, part II : $\mathrm{AUSM}^{+}$-up for all speeds. Journal of Computational Physics 2006; 214(1):137-170, doi:10.1016/j.jcp.2005.09.020.

34. Girimaji S. Assumed $\beta$-pdf model for turbulent mixing: Validation and extension to multiple scalar mixing. Combustion Science and Technology 1991; 78(4-6):177-196, doi:10.1080/00102209108951748.

35. Yoon SH, Kim C, Kim KH. Multi-dimensional limiting process for three-dimensional flow physics analyses. Journal of Computational Physics 2008; 227(12):6001-6043, doi:10.1016/j.jcp.2008.02.012.

36. Van Leer B. Towards the ultimate conservative difference scheme. V. A second-order sequel to godunov's method. Journal of Computational Physics 1979; 32(1):101-136, doi:10.1016/0021-9991(79)90145-1.

37. Sweby PK. High resolution schemes using flux limiters for hyperbolic conservation laws. SIAM Journal on Numerical Analysis 1984; 21(5):995-1011.

38. Simsont YH, Gerlinger P. High order numerical simulation of the thermal load on a lobed strut injector for scramjet applications. International Journal for Numerical Methods in Fluids 2015; submitted.

39. Ko NWM, Kwan ASH. The initial region of subsonic coaxial jets. Journal of Fluid Mechanics 1976; 73(2):305 332, doi:10.1017/S0022112076001389.

40. Oschwald M, Naclerio S, Yang B, Cuoco F. Experimental investigation of flame stabilization in coaxial $\mathrm{LOX} / \mathrm{H}_{2}$ and $\mathrm{LOX} / \mathrm{CH}_{4}$ spray flames. Proceedings of the 22nd European Conference on Liquid Atomization and Spray Systems - ILASS 2008, 2008 